JAKUB ALEJSKI

Faculty of Anthropology and Cultural Studies Adam Mickiewicz University in Poznań, Poland

\section{Prototype worlds of video games}

\begin{abstract}
Aвstract. Alejski Jakub, Prototype worlds of video games. "Images" vol. XXIX, no. 38. Poznań 2021. Adam Mickiewicz University Press. Pp. 33-42. ISSN 1731-450X. DOI 10.14746/i.2021.38.02.

In this paper the author analyzes the phenomenon of prototype worlds - synthetic environments of simulators, video games and other types of software - used to conduct experiments at the level of user sensorium, environmental physics and social design. The author presents the evolution of the concept, beginning with Buckminister Fuller's World Game project, moving through media experiments in the field of game design, and finally presenting contemporary applications (such as a drone pilot training project for the U.S. Air Force) and their implications.
\end{abstract}

KEYwords: Game studies, synthetic ecologies, media design, Buckminister Fuller, prototype worlds

In 1967, Richard Buckminster Fuller presented the World Game project for Expo ' 67 in Montreal; it consisted of creating a prototype model of the world in which, using scientific models concerning economy, ecology and sociology, it would be possible to conduct experiments on solving the various problems of the modern world. The project was to take the form of a game in which participants would implement various experimental concepts in order to "make the world work, for 100\% of humanity, in the shortest possible time, through spontaneous cooperation, without ecological offense or the disadvantage of anyone." [1] World Game was ultimately supposed to function as a computer-powered game that would allow operations in a prototype world. The aim of this article is to identify this particular kind of video game world - the prototype world - and the strategies for entering into a relationship with it, using experimental activities. According to Carlye Lauf, Daria Kotys-Swartz and Mark Rentschler, the prototype is a "complex and dynamic artifact perceived as active tool used in the design process and as influential actor impacting social situations." [2] Following this reasoning, the prototype world is both a tool used "to actively carry out intentional functions and actor shaping social situations and networks, either intentionally or unintentionally" [3].

Fuller's prototype world was not fully realized for a long time. The World Game Series: Document One from 1971 stated that "the World Game has not yet, in truth, been played" [4]. The most important factor
[1] Buckminster Fuller Institute website: <https:// www.bfi.org/about-fuller/big-ideas/world-game>, accessed: 24.01.2021.

[2] C.A. Lauff, D. Kotys-Schwartz, M.E. Rentschler, What is a prototype? What are the roles of prototypes in companies?, "Journal of Mechanical Design" 2018, vol. 140, no. 6 .

\section{Images}

vol. XXIX/no. 38

Poznań 2021

ISSN 1731-450X

\section{Introduction}

[3] Ibidem.

[4] R. Buckminster Fuller, The World Game: Integrative Resource Utilization Planning Tool, Carbondale 1971, p. x. 
preventing gameplay was the lack of an available computer system capable of handling a huge mathematical model. The second reason is related to the requirement of the application's "logic to ask the right questions." [5] The problems identified in the document allow us to highlight two strategic goals of the project. The first was to be achieved using design science methodology in the game, "to explore for ways to make it possible for anybody and everybody in the human family to enjoy the total earth without any human interfering with any other human and without any human gaining advantage at the expense of another." [6] The second goal is related to the formation of the aforementioned logic, according to which the problems of the world should be considered in a global perspective, rejecting "local focus" in favour of treating the entire "Spaceship Earth" as a relevant unit of analysis. Following Buckminster Fuller's concept, in this article, the effects of experimenting within the prototype worlds have been divided into two categories: firstly, the data obtained as a result of the experiment on the behaviour of the model under certain conditions (the effect of using a prototype world as a tool); the second is the impact on the way the user perceives and/or interprets reality as a result of experimental activities (the effect of interacting with the prototype world as an actor).

It is worth noting that thinking about the user's actions in the category of an experiment was present not only in scientific projects using the medium of video games, but also in the context of commercial productions. Video game designer Will Wright describes his interaction with the 1981 flight simulator as follows:

The first thing I did was I went in and started exploring the behaviour space. Trying all the different things with the airplane. What happens if I go straight up? How far can I go? What happens if I crash? What happens if I do this that and the other? So I could carry out experiments in this world. And in running those experiments I could get a more accurate view of what the internal model was. So it's kind of a scientific process. It's kind of a "hypothesize, experiment, change your hypothesis” type cycle that was going on.[7]

The above example shows that thinking about synthetic worlds in the category of a prototype has been present from the very beginning of this medium's functioning in media culture. This seems to be a natural consequence of the video game development process; each game world and the system of rules that governs it must be repeatedly checked and tested, not only for possible errors, but also to evaluate the generated user experience. From this perspective, the concept of the world as a prototype is one of the fundamental aspects of this medium. Not every game world activity is an experiment, nor is every game

[5] Ibidem.

[6] Ibidem, p. 89.
[7] C. Pearce, Sims, BattleBots, Cellular Automata God and Go. A conversation with will wright by celiapearce, "Games Studies" 2001, <http://www.gamestudies. org/0102/pearce/>, accessed: 24.01.2021. 
world a prototype world. The experiment requires a hypothesis and its subsequent verification based on the obtained data. The basic condition for the functioning of the prototype world is its potential to create emergent gameplay. It occurs when a player's actions result in a second order of consequence that the development team did not predict and the game behaves in a rational but unplanned way. It allows players to solve game problems by using strategies that were not envisaged by the designers. The functioning of a world designed in such a way is a direct consequence of the implementation of the rules of preserving individual elements, not a scenario predicted and designed by the developer. Only with the assumption that the system's reaction to the user's experimental behaviour is a logical consequence of the functioning of a given world is it possible to perceive this world in terms of a prototype. Only when the user decides to undertake experimental activities in the emergent world of the game does the prototyping process take place. This means that prototyping requires the player to act intentionally, the final effect of which will be to learn the cause-effect sequences that define one or more aspects of the gameworld. The scope of the possible performance of the world's prototype potential depends to a large extent on the design of this world. In the following part of the article, we will present three aspects of the prototype worlds commonly found in experiments: sensorium, physics and social prototyping.

In the prototype worlds of video games, the potential for experimentation takes place at the level of the sensorium, always shifting, dynamic „subject's way of coordinating all of the body's perceptual and proprioceptive signals as well as the changing sensory envelope of the self.' [8] Perception of time, spatial orientation, and interacting with the environment require the user to use other areas of his sensorium than in the physical world. Modern computer systems (both personal computers and consoles) seemingly offer the user a very limited set of senses that they can use to collect and interpret data from the gameworld. In most cases, it is sight (image on the screen, possibly controller light messages), hearing (environmental sounds, interface and music), and in some cases, touch and body position (controller vibration and its orientation in space). The user is deprived of taste and olfactory stimuli, sense of speed, balance, temperature, pain and many others. In such a situation, the prototyping process of the user's sensorium may follow one of two directions. The first one answers the question of how to design the world to minimize the number and variety of stimuli, transmitting all the necessary information using a limited sensorium; while the second answers the question of how to design a stimulus communication system using technologically-mediated synaesthesia[9]

[8] C.A Jones, Mediated sensorium, [in:] Sensorium: Embodied experience, technology, and contemporary art, ed. C.A Jones, Cambridge 2006.

\section{Prototyping} the sensorium
[9] K. Suslick, Synesthesia in science and technology: More than making the unseen visible, "Current Opinion in Chemical Biology" 2012, vol. 16, no. 5-6, pp. 557-563. 
in such a way that the user's sensorium is extended with new strategies for interpreting reality.

In the first case, the design of the game world completely eliminates difficult-to-transmit the sensual stimuli; for example, the sense of vertical orientation is eliminated by reducing the space of the game world to two dimensions. In two-dimensional space, all objects are on one plane, so orientation in the up-down axis loses any meaning, as it has no consequence. In the latter case, it is necessary to develop sensory strategies that the user needs to know, understand and assimilate. High speed blur, red flashes from the pain source, the controller shaking while driving, information on the interface to orientate yourself in time and space (such as calendar, clock, map or compass) - all these messages are intended to translate stimuli from inaccessible sense to sight, hearing or touch. The user must recognize new forms of sensory communication through experimental activities. Their situation is special - they are in two realities simultaneously, each of which, due to the rules governing it, requires different cognitive strategies. Agnieszka Jelewska points out the constant process of synchronizing the human sensorium with reality, stating that "the human sensorium is formatted at every moment, in every act of perception, and most importantly - in interaction with the surrounding space, with objects and objects that form us at the same time." [10] Thanks to the need to suspend the strategy of perceiving and interpreting stimuli from the physical world, the user has the opportunity to adapt his sensorium to the principles of the functioning of the prototype world with which he interacts.

Prototyping the physics
The need to format the user's sensorium is particularly evident when the prototype world not only requires new sensory strategies, but is primarily designed with rules which contradict the laws of the physical world. This idea was behind the first virtual reality systems, the construction of a prototype world that would allow the visualization of physical laws which, until now, could only be represented by mathematical formulas. Ivan Sutherland, who constructed the first functional virtual reality system at the University of Utah in 1970, described his concept as follows:

For instance, imagine a triangle so built that whichever corner of it you look at becomes rounded. What would such a triangle look like? Such experiments will lead not only to new methods of controlling machines, but also to interesting understandings of the mechanisms of vision. There is no reason why the objects displayed by a computer have to follow the ordinary rules of physical reality with which we are familiar. The kinaesthetic display might be used to simulate the motions of a negative mass. The user of one of today's visual displays can easily make solid objects transparent - he can „see through matter!"[11]
[10] A. Jelewska-Michaś, Sensorium: eseje o sztuce i technologii, Poznań 2013, p. 31.
[11] I. Sutherland, The ultimate display, [in:] Multimedia: From Wagner to virtual reality, expanded edition, 
The prototype worlds of modern video games are performed by computer systems with incomparably greater computing power than those with which Fuller or Sutherland worked. For this reason, the models of these worlds can be described by many interdependent rules and procedures. Due to the great potential for creating emergent gameplay, the user's strategies involving experimentation with individual elements of the game world can take on very complex forms. At the same time, the software nature of the worlds in question makes each of them, at the basic level, a cybernetic assembly of numerical data and the procedures which manage this data. This means that the programmer who writes the code must introduce into the world of design every tiny rule that is to apply. In the case of prototyping an object in the physical world (e.g. an airplane), the designer has no control over the laws of physics, gravity, and the properties of time and space; the whole point of building a prototype is to verify the assumptions about its functioning in the physical world, such as, for example, whether the wing's lifting surface is sufficient to keep the plane in the air at a certain speed. In the case of prototyping the world of a video game, the designer not only has to introduce visible elements into it, such as territory, objects or light sources, but most of all has to define the rules governing space-time. Neither of these principles is implied; if omitted at the design stage, a principle will have no application to gameplay and subsequent emergent processes[12].

In many modern puzzle games, the core of the gameplay is based on the implementation of different rules for the functioning of spacetime in comparison to the physical world; to solve subsequent puzzles, the user must recognize these rules and then learn to use them to achieve a specific goal. An example of a prototype world that requires the reconceptualization of the perception of space is Portal,[13] a game released by Valve Software. The user's goal is to arrange elements in three-dimensional space using direct interaction (lifting objects by the avatar) or using the possibility of creating the portals - pairs of connected holes disturbing the three-dimensionality of space - that can connect any two points of a given room. The principle of the portals is simple: entering one of them causes an exit through the other. In this way, the user is able to place the lifted objects in places to which they have no access under normal conditions. The user could also carry out experiments in the prototype world unrelated to the solution of the puzzle; for example, will an object thrown into a system of portals

ed. K. Jordan, R. Packer, New York - London 2000, p. 256.

[12] There is a derogation from the need to intentionally implement every rule that governs the world of a video game, which is the use of game design engines and ready-made scripts in the design process. In this case, the designer, when deciding on a specific software, introduces to his gameworld core rule set, the content of which differs depending on the engine used. However, it is still reasonable to say that the choice of such software is an intentional decision of the designer, as is the decision to leave the core rule sets in the design.

[13] Portal (Valve Software, 2007). 
arranged one above the other accelerate endlessly, or is there a speed limit, and if so, is it identical for all objects?

Another example of introducing different physical rules is changing the way time passes in the game world. Such a situation occurs in the case of SUPERHOT,[14] a first-person shooter game in which the passage of time in the game world is directly related to the movement of the player's avatar in space or their actions. Until the user decides to perform an action, the passage of time in the game world is almost completely stopped. Such a situation requires the user to change their perception of spatio-temporal relationships; it only makes sense to make the necessary movements that will allow the user to change the situation to their advantage, while the strategies found in many other video games of constantly moving in order to make it as difficult as possible for the opponent to score a hit are completely lost.

\section{Prototyping the social}

Video game worlds can be more than just prototypes of alternative physical laws of space, time, and objects. Under certain conditions, they also allow for social prototyping. In order for the creation of such a prototype to be possible, the rules governing social relations between individual actors in the game world must allow emergent phenomena to occur. Numerous and scattered social events between actors should be the result of the relationship of certain parameters of all participants in a given event.

An example of such a designed social engine is the video game Rimworld[15], released by Tynan Sylvester, in which the gameplay is about managing a colony and colonists on a remote planet in the distant future. What makes Rimworld significantly different from other similarly-themed video games is its special story-building strategy, as Sylvester wrote on the game's official website:

It's not about winning and losing - it's about the drama, tragedy, and comedy that goes on in your colony. The game creates events like pirate raids, trader arrivals, and storms. But these events aren't random. RimWorld uses an AI Storyteller (modeled after the AI Director from Left 4 Dead) who analyzes your situation and decides which event she thinks will make the best story.[16]

The story that develops during the game is not based on previously-designed events and relationships, but each scene consists of a large number of insignificant events, which, when added together, gain significance for the whole. One of the key elements of the storytelling mechanism is the aforementioned social engine that regulates all relationships between the colonists. Its operation is based on numerical parameters that determine the mood of a given colonist, their specific physical and mental characteristics, and their relationship to all other colonists. Each action taken by the colonist influences the values of

[14] SUPERHOT (SUPERHOT Team, 2016).

[15] RimWorld (Ludeon Studios; 2018).
[16] Game official website: <https://rimworldgame. com/index.php?lang=en>, accessed: 24.01.2021. 
these parameters in a positive or negative way. At the same time, colonists' confrontations with specific features and values of parameters may lead to events defined in the rules of the game. Colonists who do not like each other will start fights, work less efficiently, or even suffer from a nervous breakdown. On the other hand, sympathetic colonists will be in a good mood to be together, and if the criteria of age and sexual orientation are met, they can initiate an affair that can develop into a permanent relationship, a painful break-up, or otherwise. The social prototype in this case are the rules according to which the social sphere of the world is built. As with the rules for world physics, only events that have been coded by the designer can appear during the game. This means that each rule regulating social relations between actors had to be precisely defined and written into the game code. For this reason, careful analysis of the game code reveals the basic rules governing the world that may not be clearly visible in emergent gameplay. This analysis became the basis of Rimworld's feminist criticism.

The case was described by Claudia Lo in "Rock, Paper, Shotgun" [17] in 2016, and later developed by Cecilia D'Anastasio on the "Kotaku”[18] website. During the analysis of the code, it was noticed that particular rules concerning relationships between women and men reproduce ideas with a clearly sexist basis. In particular, they concerned the likelihood of initiating a romance (in the case of men, it was eight times higher), the recognition of people with disabilities as always less attractive, the lack of opportunities for bisexual men (homosexual or heterosexual only) and heterosexual women (homosexual and bisexual only), as well as correlations between the age and the attractiveness of individual actors (for example, a woman who is older by more than 15 years is completely unattractive to a man, women perceive older partners as more attractive, men perceive women aged 20 to their age as the most attractive, etc.). The prototype world of Rimworld unintentionally addressed an important social problem: the perception of gender role relations in the context of video games. It provoked numerous comments, discussions, and, consequently, changes in the game code; users decided that they were not willing to accept a social world where gender relations are designed in a specific way. The experimental act (the gameplay and its analysis in Rimworld) led to questioning the previous logic of perceiving gender social relations in video games.

When analysing experimentation with the social aspect of the prototype worlds, it is worth mentioning about Edward Castronova's concept of synthetic worlds, which he defines as "an expansive, worldlike, large-group environment made by humans, for humans, and which

[17] C. Lo, How Rim World's code defines strict gender roles, "Rock Paper Shotgun" 2016, <https://www.rockpapershotgun.com/2016/11/o2/rimorld-code-analysis/>, accessed: 24.01.2021.
[18] C. D'Anastasio, RimWorld's queer women controversy, explained, "Kotaku" 2016, <https:// kotaku.com/rimworlds-gay-women-controversy-explained-1788555928>, accessed: 24.01.2021. 
is maintained, recorded, and rendered by a computer." [19] A feature that distinguishes Castronova's synthetic worlds from other prototype worlds is their potential to provide a common space for many online users. For this reason, experiments taking place in synthetic worlds more often concern the social relationships between users within the rules of a given world. Most of today's synthetic worlds are referred to as Massively Multiplayer Online Role-Playing Game and are represented on the video game market by titles such as World of Warcraft,[20] Guild Wars 2,[21] and EVE Online.[22] In particular, the latter is an interesting example of a prototype world, as in 2020, on average, 34,000 users were logged in simultaneously on a single server.[23] Such a large number of active players means that within the synthetic world, thanks to emergent processes, the economy functions in accordance with micro- and macroeconomic principles. [24] Experimental actions by users and administrators (for example, introducing alternative monetary regimes, developing adaptation and resistance strategies) provide data on social and economic responses to movements that are considered too difficult or risky in the case of the global economy.

Consequences

Experiments in the economies of the prototype worlds are not entirely free from consequences in the real world. In our EVE: Online example, there is a direct link between in-game currency (ISK) and the U.S. dollar (USD). Each user, in order to gain access to the server for 30 days, must purchase PLEX points using real world currency. At the same time, PLEX points are an item in the game world that can be purchased with the help of ISK from other users. This means that ISK currency has its own exchange rate, mediated by PLEX points and stabilized by the number of users in the economic system. This relatively simple method of valorising ISK currency leads to legally and economically unclear situations in which the user is able to, for example, "open a bank and then walk off with about 700 billion in the game's ISK currency, or close to $\$ 120,000$ in U.S. dollars if sold illegally to other players on eBay." [25]

The above-described experimental processes within prototype worlds can also have implications entirely beyond the user-video game relationship. Their potential use is recognized and applied by entities that are in no way related to the video game industry. The development of new sensory strategies by users can be stimulated and used by these

[19] E. Castronova, Synthetic Worlds, Chicago - London 2008.

[20] World of Warcraft (Blizzard Entertainment, 2004)

[21] Guild Wars 2 (ArenaNet Inc., 2012)

[22] EVE: Online (Crowd Control Production; 2003)

[23] Data for the Tranquility server for 2020, source: $<$ https://eve-offline.net/?server=tranquility $>$, accessed: 24.01.2021.
[24] E. Castronova, op.cit., pp. 182-204.

[25] C. Chambers, How virtual are virtual economies? An exploration into the legal, social and economic nature of virtual world economies, "Computer Law \& Security Review: The International Journal of Technology Law and Practice" 2011, vol. 27, no. 4, pp. $377-384$. 
entities to achieve tangible profit. An example of such a procedure can be the research conducted in cooperation with the U.S. Air Force,[26] devoted to the correlation between the time spent by the subject playing video games and the predisposition to operate and control UAVs (Unmanned Aerial Vehicles). Another aspect of this research is the analysis of decision-making competence at different risk levels. [27] The aim of the research is to search for specific candidate features for operators that will allow for shortening the training time, thus reducing its cost. In the conclusions of the research, it can be seen that "cognitive skills learned in video game play may transfer to novel environments and improve performance in UAS tasks over individuals with no video game experience" [28]. This does not mean that interacting with the prototype worlds can make the user a pilot, but it is a significant premise that extending their sensorium significantly increases the user's predisposition to this role. In this perspective, it seems justified to speculate about the possibility of shaping the prototype worlds of video games by interested entities in such a way as to consciously or unconsciously model the cognitive competences of future candidates.

The second consequence is related to the collection, analysis and use of data by third parties. As has already been pointed out with EVE: Online, the user is not the only actor conducting experiments in the prototype world. Developers collect massive amounts of data on user behaviour in order to model even more attractive product content for consumers. This thought was clearly expressed by Will Wright in the context of The Sims development strategy: „I'm trying to basically chronicle the average model that the players have made in their heads. Already it's having a huge impact on what we do with our expansion packs and the next version of the game." [29] In his considerations, Wright outlines a reality in which developers will be able to both adapt the product to the expectations of consumers, and at the same time, gradually adjust these expectations to the current policy of the company.

Performing prototype worlds - from Buckminister Fuller's World Conclusions Game and Ivan Sutherland's virtual worlds to modern video games brings with it a potentially significant social change. By experimenting, users can not only obtain data on the effects of the actions taken (thanks to the use of the prototype world as a tool), but more importantly, the relationship between the user and the prototype world, understood as an actor, leads to the user generating new strategies for using the

[26] A.R. McKinley, L.K. McIntire, M.A. Funke, 2011, Operator selection for unmanned aerial systems: comparing video game players and pilots, "Aviation, Space, and Environmental Medicine" 2011, vol. 82, no. 6, pp. $635-642$.

[27] J.M. Wheatcroft, M. Jump, A.L. Breckell, J. Adams-White, Unmanned Aerial Systems (UAS) operators' accuracy and confidence of decisions: Professional pilots or video game players?, "Cogent Psychology" 2017, no. 4.

[28] A.R. McKinley, L.K. McIntire, M.A. Funke, op.cit. p. 1.

[29] C. Pearce, Sims, BattleBots, Cellular Automata God and Go. A Conversation with Will Wright by Celia Pearce, "Game Studies", <http://www.gamestudies. org/0102/pearce/>, accessed: 24.01.2021. 
sensorium and new logics of conceptualizing reality. It is also worth remembering that this significant social change can also be used to design a society in accordance with the interests of groups and entities completely independent of users. For this reason, analysing contemporary prototype worlds and the experiments undertaken within them is one of the most important challenges facing modern game studies.

B I B L I O G R A P H Y
Buckminster Fuller R., The World Game: Integrative Resource Utilization Planning Tool, Carbondale 1971

Castronova E., Synthetic Worlds, Chicago - London 2008

Chambers C., How virtual are virtual economies? An exploration into the legal, social and economic nature of virtual world economies, "Computer Law \& Security Review: The International Journal of Technology Law and Practice" 2011, vol. 27, no. 4, pp. 377-384

D’Anastasio C., RimWorld's queer women controversy, explained, "Kotaku" 2016, <https://kotaku.com/rimworlds-gay-women-controversy-explained1788555928>, accessed: 24.01.2021

Jelewska-Michaś A., Sensorium: eseje o sztuce i technologii. Poznań 2013

Jones C.A., Mediated sensorium, [in:] Sensorium: Embodied Experience, Technology, and Contemporary Art, ed. by C.A. Jones, Cambridge 2006, pp. 5-49

Lauff C.A., Kotys-Schwartz D., Rentschler M.E., What is a prototype? What are the roles of prototypes in companies?, "Journal of Mechanical Design" 2018, vol. 140 , no. 6

Lo C., How Rim World's code defines strict gender roles, "Rock Paper Shotgun" 2016, <https://www.rockpapershotgun.com/2016/11/02/rimorld-code-analysis/>, accessed: 24.01 .2021

McKinley A.R., McIntire L.K., Funke M.A., Operator selection for unmanned aerial systems: comparing video game players and pilots, "Aviation, Space, and Environmental Medicine" 2011, vol. 82, no. 6, pp. 635-642

Pearce C., Sims, BattleBots, Cellular Automata God and Go. A Conversation with Will Wright by Celia Pearce, "Games Studies", <http://www.gamestudies.org/0102/ pearce/>, accessed: 24.01.2021

Suslick K.S., Synesthesia in science and technology: More than making the unseen visible, "Current Opinion in Chemical Biology" 2012, vol. 16, no. 5-6, pp. 557-563

Sutherland I., The ultimate display, [in:] Multimedia: From Wagner to Virtual Reality, Expanded Edition, ed. K. Jordan, R. Packer, New York - London 2000

Wheatcroft J.M., Jump M., Breckell A.L., Adams-White J., Unmanned Aerial Systems (UAS) operators' accuracy and confidence of decisions: Professional pilots or video game players?, "Cogent Psychology" 2017, no. 4

Guild Wars 2 (ArenaNet Inc., 2012)

EVE: Online (Crowd Control Production; 2003)

Portal (Valve Software, 2007)

RimWorld (Ludeon Studios; 2018)

SUPERHOT (SUPERHOT Team, 2016)

World of Warcraft (Blizzard Entertainment, 2004) 